\title{
The Structure and the Means of Expression in the Conversational Speech of Women in the Southern Region of Albania
}

\author{
Msc. Aida Lamaj \\ "Eqrem Çabej"University, Department of Foreign Languages,"Eqrem Çabej" University, Albania \\ Email address: aida_lamaj@yahoo.it \\ Msc. Floriana Pango \\ "Eqrem Çabej"University, Department of Foreign Languages, "Eqrem Çabej" University, Albania \\ Email address: floriana_mera@yahoo.it
}

\section{Doi:10.5901/ajis.2016.v5n1p167}

\section{Abstract}

\begin{abstract}
The aim of this article is to give a modest contribution in the description of the structure and the means of expression of the feminine speech in the Southern region of Albania. Communication between the sexes has been an area of interest of sociolinguists that have suggested that we come from different planets, that we have different ways of talking, thinking, different brain organizations, etc. Research on language and gender has increasingly become research on gender and discourse. A movement toward the study of language within specific situated activities reflects the importance of culturally defined meanings both of linguistic strategies and of gender. The study of gender and discourse not only provides a descriptive account of male/female discourse but also reveals how language functions as a symbolic resource to create and manage personal, social, and cultural meanings and identities. We have analyzed the everyday language of men and women, in everyday situations as well as in the public communications, in the cities and in the small villages. We have used some examples from the artistic literature of feminine writers of this region. The present paper tries to point out some of the male/female differences in communication and to find the answer to the question: in which way men and women in this region speak differently?
\end{abstract}

Keywords: feminine speech, gender, difference, Southern region of Albania, discourse

\section{Introduzione}

"Noi tutti sappiamo di essere unici, ma tentiamo di considerare gli altri come membri di qualche gruppo. Questo è un processo naturale che parte dal nostro bisogno di guardare il mondo nei suoi elementi per capirlo meglio" (Tannen, 1991). Per studiare a fondo le differenze che corrono tra il linguaggio degli uomini e delle donne, sono stati usati diversi metodi: Jespersen (1922) e Lacoff (1975) hanno fatto ricorso all'intuizione e alle loro impressioni, un procedimento normale negli studi del Novecento; più tradizionale appare il metodo dell'osservazione diretta della comunicazione spontanea; altre volte sono stati usati dati di laboratorio per mantenere intatte alcune variabili, le quali, in altre condizioni, avrebbero potuto inficiare il risultato dell'indagine; infine, ci si è rivolti anche alle valutazioni dei parlanti sul parlato proprio e altrui. Ogni metodo possiede dei pregi e anche dei difetti che vanno attentamente valutati in rapporto all'oggetto della ricerca. Per esempio, il metodo basato sui dati di laboratorio ci permette di isolare il fattore "genere" in modo tale che le altre variabili, come lo status e il ruolo esercitato nella situazione comunicativa dall'emittente e dal ricevente non influiscano sulla frequenza delle variabili prese in considerazione; dall'altra parte, le condizioni riprodotte nel laboratorio sono artificiali e possono influenzare il risultato. È inoltre tutt'altro che facile individuare le forme linguistiche che presentano una forte dipendenza da particolari situazioni comunicative e altresì alcuni elementi conversazionali tipici del linguaggio spontaneo. Questo costituisce uno dei problemi più rilevanti nell'interpretazione dei dati che provengono dalla comunicazione nella vita reale.

In effetti, molte variabili indipendenti riferite agli interlocutori, oltre allo status e al ruolo, l'età, il livello d'istruzione, si intrecciano alle situazioni comunicative. Sarebbe necessario uno studio più approfondito che ci permetta di capire dove si verificano le differenze attribuite al comportamento e come le diverse variabili di tanto in tanto influiscano l'una sull'altra.

Le cause della diversità nel comportamento linguistico sono state ricercate non tanto nelle differenze biologiche, ma nelle situazioni socio-culturali (Kramarae, 1981:31).

"La conversazione si formalizza in rapporto alla vita dell'individuo, al suo lavoro e alla sua attività" (Lloshi, 1999: 
321). La donna è un membro importante in una società che genera, struttura e trasmette il suo linguaggio con mezzi e forme diversi. In effetti la nostra è una società in cui le interazioni comunicative seguono un insieme di regole ben stabilite (Pango,1997:102). Nonostante l'ambizione degli uomini di dimostrare di riuscire a fare tutto o di cavarsela in tutto e il tentativo delle donne di provare a se stesse di essere in grado di attrarre gli altri (From, 2006:122), tra i due generi è necessario il libero scambio dei pensieri 0 il confronto sulle problematiche relative alla vita quotidiana, alla famiglia 0 al lavoro. Uomini e donne costruiscono i loro rapporti grazie alle pratiche e alle interazioni linguistiche, cioè grazie alla comunicazione. Ma la lingua attuata nel parlato non ha un aspetto uniforme. Così come la società si basa su una sua gerarchia, anche la lingua si articola e si accorda a questa gerarchia. Savoia (2009:24) nota che: "Le variabili linguistiche correlate a sesso, età, appartenenza etnica, classe sociale soggette a variazione stilistica in rapporto alla situazione, sono dette marcatori; si tratta di realizzazioni di cui il parlante può prendere coscienza e che comunque sono regolarmente attivate dai parlanti. Gli indicatori sono variabili che non hanno alternanza stilistica e corrispondono a differenze linguistiche che rappresentano aspetti fissi dell'identità dei parlanti, collegate a differenze sociali che non si modificano in rapporto alla situazione. Gli stereotipi sono realizzazioni esplicitamente riconosciute dalla società, generalmente oggetto di stigmatizzazionee spesso correlate a pregiudizi o comunque ad un sistema simbolico rigido e preconfezionato."

Oggi è ammesso da tutti che ogni parlante (anche quello femminile) possieda non solo un repertorio linguistico, il cui funzionamento si collega a norme sociali precise, ma un arsenale di strategie linguistiche naturali e acquisite, collegate fortemente all'identità sociale di ogni individuo (Baylon, 1996:220).

Gli eventi linguistici legati al destino, ai sentimenti, al mondo personale e professionale del sesso femminile si rendono chiari grazie agli atti comunicativi. Nella linguistica europea non è stata sostenuta solo l'idea che l'atto comunicativo sia tanto importante quanto la lingua stessa, ma su di esso sono stati effetuati studi diversi. Così, nel volume "La linguistica e la poetica", R. Jakobson, individuando sei fattori dell'atto comunicativo, facilita la strada agli altri studiosi per argomentare che "le repliche della conversazione possono essere descritte linguisticamente, quando si confrontano le interazioni e le sue conseguenze tra i parlanti e gli interlocutori e la situazione in cui si effetua la comunicazione".

La strada avviata dalla linguistica paragonata sullo studio dell'atto verbale, è stata seguita da antropologi e etnologi. Di conseguenza, è nata la branca dell'etnografia del parlato che mira a una descrizione dettagliata degli atti o dei comportamenti linguistici. Uno degli studiosi che ha avuto un ruolo di spicco nella scoperta e nella descrizione del comportamento verbale è Hymes, che non considera le informazioni casuali, ma grazie a uno studio attento e alle ricerche condotte, afferma che "le funzioni e i modelli del parlato cambiano da un gruppo all'altro, o che il parlato si trasforma in un'unità di valutazione del comportamento e dell'educazione dell'individuo (Hymes, 1973), dove i più grandi consigliano, rimproverano, incoraggiano ed emancipano i più giovani, le generazioni antecedenti trasmettono il modello degli usi conversazionali all'interno di una comunità".

Lo sviluppo delle ricerche sul linguaggio femminile è stato influenzato anche da fattori sociali, come la percezione della famiglia come una struttura elementare, la considerazione della vita individuale come un teatro importante dell'esistenza. II ruolo del "genere" è considerato come il più importante del comportamento sociale.

\section{Gli Scopi Comunicativi del Linguaggio della Conversazione Femminile}

Il sesso femminile realizza i suoi scopi secondo il postulato: la vita è una battaglia per conservare la vicinanza con gli altri ed evitare l'isolamento (Hudson, 2002:159). In effetti alcuni linguisti hanno perfino parlato di discriminazione linguistica delle donne. Secondo Lacoff, "...l'esperienza linguistica discriminatoria delle donne appare in due forme: nel modo in cui le donne si sono abituate ad usare il linguaggio e nel modo in cui il linguaggio generalmente le tratta".

Tenendo presente le tendenze, le capacità e i ruoli che le donne occupano nella società odierna europea, le correnti femministe hanno mirato a status più alti. Le donne nelle regioni del Sud dell'Albania hanno desiderato una posizione pari all'altro sesso e hanno lottato per guadagnarla, il che trova espressione anche nei modi di dire della cultura popolare di questa regione: "Dielli kur bie, bie për të gjithë dhe jo vetëm për një". II gruppo femminile ha costruito la propria personalità contrapponendosi al sesso maschile - sempre in ricerca di prestigio e di interdipendenza - che da sempre, ha considerato le donne come un gruppo inferiore, etichettato come "bukuri e mënt për dhi" (Sazani, 1999). In effetti, nella comunicazione pubblica e privata le diversità sono numerose, dall'intonazione della voce fino agli altri meccanismi linguistici (Kreitner; Kinicki, 2008:316).

Secondo il grammatico americano Chomsky (1965), ogni essere umano è munito, da quando nasce, della capacità di usare una lingua verbale. Indipendentemente dal codice, è importante affermare che la capacità di parlare si 
trasforma in utilità solo quando è impiegata per realizzare una serie di atti comunicativi. La conversazione, come una delle forme principali della comunicazione, accanto ai mezzi linguistici, si arricchisce anche di elementi extralinguistici.

Secondo Tannen: "La vita di ogni persona è vissuta come un insieme di conversazioni". È ovvio che la conversazione è un'attività che prevede la presenza di due o più persone con dei rapporti linguistici e sociali, con dei legami dinamici e interattivi, dei segnali e dei simboli paralinguistici che influenzano la generazione e la trasmissione dei messaggi cioè essa si fonda sul "principio della collaborazione" ed è finalizzata alla realizzazione di questo scopo. "La conversazione presuppone la presenza degli interlocutori. In alcuni casi l'interlocutore non è fisicamente presente, ma immaginario" (Lloshi, (1999:322).

Secondo Lacoff, nella maggior parte delle lingue del mondo, non esistono leggi particolari sull'uso della lingua da parte degli uomini e delle donne, ma piccoli elementi che governano il loro linguaggio.

\section{Gli Stili della Conversazione Degli Uomini e delle Donne}

"La conversazione è un tipo di testo con le proprie regole, il cui senso delle frasi si costruisce dall'interazione comune delle persone che parlano" (Lloshi, 1999:321).

Le ricerche intorno alla lingua e al genere si sono concentrate anche sugli stili di conversazione delle donne e degli uomini. L'opinione dei linguisti e quella comune li ha definiti come completamente diversi l'uno dall'altro sotto diversi aspetti.

Deborah Tannen (1984), ha individuato l'esistenza di due stili di conversazione profondamente diversi: uno stile di forte coinvolgimento (preferito dalle donne) e uno stile di forte indifferenza (preferito dagli uomini), che caratterizzano anche il comportamento nella comunicazione di ogni parlante

Usando la lingua per esprimere l'adesione al gruppo, la solidarietà e i rapporti stretti tra gli interlocutori in una conversazione, le donne generalmente si impegnano in quello che la Tannen (1990) ha descritto come "il rapporto della conversazione, nel quale il discorso simultaneo si usa per costruire relazioni o rapporti. La Tannen (1991) ha notato che "per i maschi, la conversazione è un modo di negoziare lo status all'interno del gruppo, di impedire agli altri l'esclusione dal gruppo e di conservare la propria indipendenza".

Le donne, invece, usano la conversazione per scambiare segreti e intimità; parlare è alla base dell'amicizia, così, essere amiche strette significa sedersi e parlare. Per i ragazzi, fare attività fisica e, più in generale, fare le cose insieme è fondamentale. Solo sedersi e parlare non costituisce un elemento fondamentale per l'amicizia. Essi si legano in amicizia solo con le persone con le quali eseguono diverse attività.

Sempre secondo la Tannen (1991), gli uomini non capiscono le numerose modalità in cui le donne usano la conversazione per avvicinarsi agli altri. Una di queste modalità è anche "la discussione riguardo ai problemi". La studiosa afferma che "per le donne l'essenza di un rapporto è il parlare intorno ai problemi. lo racconto a te i miei problemi, tu mi racconti i tuoi, così ci avviciniamo. Gli uomini considerano le discussioni sui problemi come una richiesta di consiglio, perciò rispondono con una soluzione. In questo caso, quando un uomo offre un'informazione di questo tipo, la donna capisce che lui, probabilmente, sta cercando di ridurre il suo problema o di cancellarlo completamente".

Analizzando in modo generico il linguaggio della conversazione femminile nelle regioni del Sud, notiamo che il laboratorio psico e sociolinguistico della bambina di fronte ai giocattoli, alle feste religiose e familiari; della madre di fronte al bambino che nasce e al quale dà un nome, e che fa crescere fisicamente e psicologicamente, facendolo stare in piedi e cantandogli ninnenanne; della ragazza che ama 0 si sente sola; della donna non protetta che affronta la mentalità patriarcale; della madre che si sente felice per il fatto che la figlia e il figlio finalmente sono cresciuti; della sognatrice che di fronte ai maschi cerca di guadagnare meriti morali e professionali; della suocera che durante la trasformazione del figlio da uomo in marito, si lamenta delle nuora ecc., contiene scopi e priorità diverse.

Le donne, nel ruolo di prime maestre, usano espressioni che socialmente considerano più utili. II loro arsenale comunicativo contiene il monologo e la comunicazione silenziosa e senza parole. Grazie a tutti questi elementi trasmettono l'amore, le coccole, la dignità femminile, la saggezza materna e linguistica ecc.

Così la madre trasmette la gioia della possibilità della riproduzione, l'orgoglio di aver dato alla luce un maschio 0 una femmina. La sua affettuosità richiama l'arrivo di un dolce sonno, trasmette il desiderio e gli auguri per il figlio/la figlia, per la loro crescita, come: hidhe, hidhe këmbën/të gëzosh nënën ecc.

Nelle ninnenanne, come artefice delle belle parole accompagnate dagli sguardi e dalle coccole, la madre diventa non solo "il rifugio e il sonno" dei bambini (Beauvoir, 2002), ma anche la codificatrice linguistica delle feste familiari che riguardano la nascita di un bambino. Insieme con gli altri, nella tradizione della bollitura dei chicchi di grano o di mais, nel portare l'acqua dalla fonte per fare il bucato, nell'uscita del primo dente, nei primi passi del bambino ecc., essa trasmette 
gli auguri più dolci.

II sesso femminile ha sempre voluto comunicare con qualcuno. Dal ruolo assegnatole dall'organizzazione della famiglia o della tribù, nelle cerimonie delle feste tradizionali e religiose come ragazza, fidanzata, donna incinta, puerpera, cognata, suocera, nascono molte parole, saluti, auguri, maledizioni ed espressioni fraseologiche. Grazie al suo modo di dialogare che porta anche variazioni nel linguaggio, si trasmettono i cambiamenti improvvisi dei codici linguistici.

All'interno della società il sesso femminile realizza anche scambi interculturali propri (Shkurtaj, 2001:195). Prima di tutto, la donna appare come formulatrice degli augùri. Le espressioni linguistiche di auguri, usate dalle donne della regione del Sud, sono in rapporto con le diverse situazioni in cui vengono formulate. Così le donne (la madre, la sorella) trasmettono durante la cerimonia delle nozze, gli auguri per il futuro sposo, per il fratello dell'"odës së qelqtë".

È interessante notare che la parlante albanese del Sud, grazie agli stimoli affettuosi e alle emozioni, qualche volta rompe le regole di una situazione formale.

In altre situazioni, tramite un linguaggio scurrile o formule di maledizione, ella esprime allo stesso tempo emozioni negative forti e qualche volta socialmente rischiose (Hudson. 2002 :24).

\section{Le Regole e le Procedure Conversazionali nel Linguaggio Femminile}

La società femminile si stratifica secondo l'età, la professione, la religione, l'istruzione e la sua emancipazione. Come membro di una società che dopo gli anni ' 90 ha avuto uno sviluppo multidimensionale, la donna albanese ha cercato una norma negli interscambi sociali e linguistici, usando i turni nella conversazione, rispettando la regola per cui quando una persona parla gli altri stanno zitti, permettendo le pause brevi (Pango,1997:103) ecc. La conversazione, come attività interpersonale, sociale, oltre alle regole astratte, grazie alle quali si costruiscono le frasi, presuppone anche regole relative al patrimonio culturale e situazionale. Così, gli interlocutori devono possedere una competenza psico-sociale che permetta di adattarsi al tema della conversazione, all'ambiente, all'organizzazione della società, alle regole non scritte, sulle cui basi ogni società assicura il proprio progresso. "Tutte le situazioni suggeriscono una comprensione sociale sicura tra le persone di un gruppo, le quali capiscono che vi appartengono e possono agire usando elementi diversi per escludere i non membri" (R. Lacoff).

II collettivo linguistico femminile, dispiegando i suoi pensieri sulla vita quotidiana, raccontando storie divertenti tra amiche ecc., ha avuto ed ha dei riti che devono essere eseguiti e un tipo di autocontrollo nel parlato (Zhugra, 2001:3031), cioè, regole sull'organizzazione della conversazione, sui temi che si discutono, sulla scala dell'adattamento della lingua alla norma standard.

Nessuno, meglio del sociologo Erving Goffman, ha capito la natura rituale della vita quotidiana e il ruolo che esercita il genere nell'organizzazione dei nostri riti quotidiani. Nel suo articolo "The arrangement between the sexes", Goffman si è soffermato in quello che tentiamo di chiamare fenomeno "relativo al genere", quando, in effetti, intendiamo "fenomeni relativi alla classe del genere". Alla base del linguaggio femminile c'è una regola universale: quando qualcuno parla, l'interlocutore ascolta e viceversa. La Tannen, partendo da questo carattere rituale dell'interazione, ha cercato di analizzare la conversazione. Secondo lei, quando cresciamo in una cultura, impariamo a fare le cose come le fanno le persone apprezzate da noi, così la maggior parte dei nostri discorsi e delle nostre decisioni diventa automatica.

Le conversazioni femminili nella regione del Sud, come una delle attività sociolinguistiche, hanno una struttura che comprende alcuni parametri:

1- il saluto;

2- la creazione di un rapporto o la dichiarazione dei ruoli;

3- il particolare compito che si articola in alcuni episodi;

4- la presa di una posizione secondo il ruolo prestabilito;

5- il saluto finale.

In questo linguaggio, ancora non studiato e trattato sufficientemente, si scambiano informazioni, si mira a un apprendimento ed insegnamento dai gruppi di status superiore. Sono ben determinati gli ambienti e le cerimonie a cui a queste attrici taciturne è permesso partecipare. Secondo la percezione tradizionale, la donna non doveva dare nell'occhio. I suoi atteggiamenti e il suo parlato si legavano più agli elementi paralinguistici, agli eufemismi ed ai parametri esteriori, che agli scambi di pensiero, 0 alla considerazione della dignità e del prestigio comunicativo (Zhugra, 2001). Così, negli scambi linguistici, appariva come parlante passiva. A lei più degli altri non si permetteva di parlare liberamente, senza badare alle regole. Nella famiglia del marito, come "proveniente" da un'altra famiglia, doveva seguire la regola: "Në këtë shtëpi këndon kokoshi" ("in questa casa comanda il marito"), accordarsi ai vizi della suocera e ai capricci del marito, essere ospitale, accettare e rispettare le idee e le decisioni del marito. 
Nelle conversazioni all'interno del gruppo, la donna concedeva l'apertura della conversazione, all'ospite 0 alla sua invitata, la quale da parte sua, nelle occasioni più formali, doveva inizialmente rivolgersi alla più anziana di quel gruppo:

"E ku vete moj Hano kështu mëngjes mëngjes? Pse nuk na je dukur fare? Pse s'vjen kurë këtej për ujë? Ne jemi gjitome dhe nga një herë s'prish punë" (Kokalari, 1944:17).

Il codice del comportamento femminile cercava l'espressione giusta e la quantità corretta del parlato. L'ospite (donna) doveva fare quanto le veniva permesso, senza vantarsi, ma consentendo anche alle altre di esprimersi.

Le donne hanno mirato alla creazione di un ambiente ospitale per gli amici. Esse, come parte di una famiglia rinomata, preparavano quello che si chiamava "la stanza della sfilata" (Zhugra, 2001:131), in cui venivano esposti davanti agli ospiti gli oggetti più preziosi della famiglia, simbolo concreto della sua grandezza e del suo valore e conservava la tradizione che vedeva aumentare la sua dignità di oratrice in rapporto al prestigio del marito.

Nei funerali queste regole diventano precise. In questi casi vengono chiamate le più anziane, che hanno come compito quello di dirigere le conversazioni; sono loro che trasmettono messaggi sconvolgenti, stando tutta la notte vicino al corpo del defunto. Esse, più che piangere, trasmettono la forza dei propri sentimenti tramite espressioni come: mu' nxi zemra, m'u drodh dora, më ngarkove rëndë, më prishe kurorën; o imzot, e zeza, ngrihu; o vëlla xhevapi; qani o lisat në brinjë ecc. In questi casi possono allontanarsi dall'atmosfera triste, parlando dei problemi, delle malattie o dei casi somiglianti ecc.

Le conversazioni familiari, come parte del rito dell'accoglienza dell'ospite, si accompagnano alla concessione del posto d'onore il quale, sia in occasioni felici che in quelli tristi, viene lasciato alla consuocera (la madre della nuora/del genero) che spesso ha il prestigio di iniziare e di concludere una conversazione.

Nelle conversazioni meno formali, tra due o più individui, le donne sono più sciolte degli uomini, sia per quanto riguarda i temi, sia per il lessico usato: parlano di tutto, fanno complimenti e nutrono la conversazione (Marinesku, $2002: 73)$.

Comunque, sono le più anziane che dirigono il discorso, il loro giudizio si considera il più corretto. Alle più anziane si permette di stuzzicare le più giovani. Durante queste conversazioni, sembra che si rompano, in qualche modo, anche i turni della presa di parola, aumentano le interruzioni e spesso gli elementi prosodici, come l'intonazione o i gesti, aiutano gli interlocutori a ordinare le parole: Pijtin kafenë dhe teto Haxhua ziente na brënda. Sa ta thosh të shkretën fjalë. $\mathrm{E}$ pastaj s'i dilte fjala nga goja. Ç'pësoi kështu?...

"-Ruho i tha kjo, "kam një fjalë për të thënë, po të keqen këtu ta thomi dhe këtu ta lëmë. E kupton dhe vetë që këta të ardhur mëngjes mëngjes, ka një mana”

- Hajli qoftë moj Haxho. Urdhëro e folë u përgjigj teto Ruhua, këtu e thomi dhe këtu e lëmë

-Të thom Ruho dhe hop luaiti na vëndi teto Haxhua...

...-Mirë thua moj Haxho... po ja... "zuri të fliste teto Ruhua...

-Lermë Ruho të sos fjalën dhe i ra në krahë.( Kokalari, 1944:17).

Le conversazioni più affettuose sono quelle del pomeriggio, trascorse magari accanto a un caminetto, bevendo un caffé e leggendo la tazzina. Qui si parla di problemi che si riferiscono al passato, in cui i sottocodici illustrano i messaggi del tempo andato, si analizza un sogno o un desiderio non realizzato e si danno le sanzioni sociali. In questi momenti di libertà, si loda 0 si rimprovera l'altro sesso, si arricchisce il racconto tramite degli aneddoti storici e divertenti. II laboratorio femminile illustra i propri pensieri con storie molto strane, in cui si incontrano molti eufemismi per indicare il demonio, la morte, le malattie ecc. Un esempio di questo tipo di comunicazione lo troviamo anche in questo brano tratto da M.Kokalari (26), una delle scrittrici più rinomate di questa regione:

Në mes të fjalëve Safua thotë "e muartë vesh...Haxhi efendiu zuri vajzën tek buri"

-“U...u...u...e kur kështu?...Tek kush e zur? Si është kjo vajzë? Kush qe krushqarja?

- "Tek djali i Mirteza agajt”... një surrat pllaçko, një hundë poçe. Përçmakë. Ç’ta shije. Të vinte anicirë t’i hidhje sitë.

- “U...u..." tha prapë Shefkua "i mirë ki djalë. Pastaj ka dhe baba nikoqir... atë nënë kapedane".

-Djali është i mirë" kërceu golë-shtrëmbëta "po nëna e mbiti. I zuri atë shahtizmeno. Se she kur në shoqe, si gjithë bota.

Pastaj moj të keqen, thonë se është e madhe. I ka vjeçët ndënë lëkurë. U nxiu sinë parai. Janë malldarë ata. Ajo është motër në di vëllezër".

- Mbaje gojën moj nikoqire...është turp të març botën në golë... "Vajza as e shastisur dhe as e mbetur brënda. Është e mir' e zgjedhurë"....

- U çke moj nikoqiere që flet kështu? Ato që më thanë, ato them dhe unë...

Çfat që pati gomarja. U...mi, çka, çoban...është! 
II dialogo femminile è uno spazio dove l'età e l'istruzione aiutano a risemantizzare la conversazione. Così le ragazze giovani non utilizzano o non conoscono le espressioni linguistiche più arcaiche, usano una lingua più vicina allo standard e alla norma letteraria (la lingua dei libri), mirando ad evitare le espressioni ironiche, pungenti o sanzionatorie.

Negli incontri più formali, come nelle scuole e nell'università, nei convegni scientifici o nelle comunicazioni di massa ecc., i gruppi e gli individui femminili mirano ad esprimersi "più formalmente", scegliendo le parole con attenzione. Nella vita di una donna, la cultura del parlato "si identifica con gli ideali della correttezza e la purezza nell'uso della madrelingua" (Kuçi, 2001:315).

\section{Conclusioni}

Concludendo possiamo affermare che tra tutti gli argomenti trattati, ciò che connota in modo particolare la conversazione femminile nella regione del Sud d'Albania, è il grado della collaborazione. Così, le donne, per natura, mirano a creare ambienti più affettuosi degli uomini e lo scopo non è la comunicazione di per sé, ma la dimostrazione dello status e la protezione dell'immagine che esse pensano di possedere all'interno della società.

Le donne nelle loro conversazioni non dimostrano una semplice reazione domanda-risposta, ma sempre e ovunque, riempiono la loro comunicazione con scambi di opinioni su temi diversi, che variano dalla gastronomia, al ricamo, dall'abbigliamento, ai mariti, dalle mestruazioni, alla crescita dei bambini e anche alla televisione, che occupa un posto importante, specialmente nella vita delle casalinghe, realizzando l'idea che le frasi e le informazioni appartengono o interessano a tutti gli interlocutori allo stesso modo (Shkurtaj, 2004:72).

Nelle conversazioni che le donne del Sud dell'Albania intrattengono tra di loro o con l'altro sesso, si rendono chiare la mentalità, la tradizione culturale folklorica di un popolo che, nonostante si trovi da secoli nel cuore d'Europa, sembra conservare ancora elementi provenienti da una realtà antica, ma in cui si rispecchia nello stesso tempo anche il desiderio di cambiare ed evolversi continuamente.

\section{Riferimenti}

Baylon, Ch. (1996). Sociolinguistique, societèe, langue et disours, Paris.

Beauvoir, S. (2002). Seksi i dytë. Tiranë.

Chomsky, N. (1965). Aspects of the theory of synthax, Cambridge Mass.

D.M.Smith, Women at work: Leadership for the next Century (Upper Saddle River, NJ: Prentice Hall, 2000, p. 26-32, in Kreitner,R., Kinicki, A. (2008). Comportamento organizzativo, Dalla teoria all'esperienza, APOGEO.

Hudson, R. (2002). Sociolinguistika, Tiranë.

Hymes, D. (1973).Verso un'etnografia della comunicazione: analisi degli eventi comunicativi, in Linguaggio e società, Bologna.

From, E. (2006). Psikoanaliza, seksi, karakteri, Tiranë.

Goffman, E. (1977). "The arrangement between the sexes," Theory and Society 4, 3:301-331, in Tannen, D., Women and Men Talking: An Interactional Sociolinguistic Approach, in Women, Men, \& Gender, Yale University Press, New Haven \& London.

Kokalari, M. (1944). Rreth vatrës, Tiranë.

Kramarae, Cheris.(1981)."Speech Styles Approach." Women and Men Speaking: Frameworks for

Analysis. New York: Newbury House.

Kuçi, S. (2001). Kultura e të folurit në televizion, Seminari XX ndërkombëtar për gjuhën, letërsinë dhe kulturën shqiptare, Prishtinë.

Lacoff, R. (1974). Language and Woman's Place, Language in Society, 2.

Lloshi, Xh. (1999). Stilistika dhe pragmatika, Toena, Tiranë.

Marinesku, A. (2002). Kodi i sjelljeve të pëlqyeshme sot, Tiranë.

Pango, Y. (1997). Psikologjia sociale, Tiranë.

Savoia, Leonardo M., Baldi, B. (2009). Lingua e società, La lingua e i parlanti, Pacini Editore SpA.

Sazani, H. (1999). E folmja e qytetit të Gjirokastrës.

Shehu, Novruz Xh. (2009). Musine Kokalari, Vepra, II, Geer, Tiranë.

Shkurtaj, Gj.(2004). Etnografi e të folurit të shqipes, Tiranë.

Shkurtaj, Gj. (2001). Onomastikë dhe etnolinguistikë, Tiranë.

Tannen, D. Women and Men Talking: An interactional Sociolinguistic Approach, Women, Men \& Gender, Yale University Press, New Haven \& London.

Tannen, D. (June 1991). How male and female students use language differently.

Zhugra, A. (2001). Rreth normave të sjelljeve gjuhësore dhe kritereve të krijimtarisë gojore, in Gjurmime albanalogjike, Prishtinë. 